\title{
Recidivas postratamiento de la lepra multibacilar
}

\author{
Gerzaín Rodríguez ${ }^{1}$, Rafael Pinto ${ }^{2}$, Carlos Laverde ${ }^{3}$, Martha Sarmiento ${ }^{3}$, \\ Angélica Riveros ${ }^{3}$, Jessika Valderrama ${ }^{4}$, Nelly Ordóñez ${ }^{4}$ \\ ${ }^{1}$ Laboratorio de Patología, Instituto Nacional de Salud; Facultad de Medicina, Universidad Nacional, Bogotá, \\ D.C., Colombia. \\ ${ }^{2}$ Asociación Alemana de Asistencia a los Enfermos de Lepra y Tuberculosis, DAHW, Bogotá, D.C., Colombia. \\ ${ }^{3}$ Programa de Control de la Lepra, Sanatorio de Agua de Dios, Agua de Dios, Cundinamarca. \\ ${ }^{4}$ Laboratorio de Patología, Instituto Nacional de Salud, Bogotá, D.C., Colombia.
}

La persistencia de bacilos viables y la monoterapia con diamino-difenil-sulfona (DDS) son los principales factores que favorecen las recidivas de la lepra. Presentamos 33 pacientes con lepra lepromatosa (LL) diagnosticada 7 a 48 años antes de la recidiva, que recibieron monoterapia con DDS durante 4 a 38 años. Veintiocho fueron tratados, además, con poliquimioterapia (PQT) irregular, no supervisada, desde 1983. Cinco sólo recibieron DDS. Éstos presentaron la recidiva entre 13 y 20 años después de suspenderla. Las recidivas se diagnosticaron por reaparición de las lesiones clínicas o por la presencia de nuevas zonas anestésicas; todas se confirmaron con la baciloscopia y, en 20 casos, por la biopsia de piel. Cuatro pacientes presentaron en la biopsia de la recidiva, lepra indeterminada (LI) y uno lepra dimorfa tuberculoide (LDT), todos con presencia de bacilos intraneurales; los demás fueron LL. Dos pacientes recidivaron, aun con PQT razonablemente supervisada. Los demás curaron con PQT supervisada. Los factores predisponentes para la recidiva fueron: monoterapia con DDS por varios años; PQT irregular con dosis inadecuadas, sin supervisión del tratamiento; abandono de la PQT, y relación inadecuada entre el paciente y el personal de salud. Las recidivas de la lepra se deben buscar en todos los pacientes colombianos con lepra multibacilar que fueron tratados con DDS solo durante años. La clínica, la baciloscopia y la biopsia individualmente o en conjunto son métodos confiables para establecer las recidivas.

Palabras clave: lepra multibacilar, recidivas de la lepra.

\section{Relapses after multibacillary leprosy treatment}

Leprosy relapses are mainly due to bacillary persistence and diamino-diphenyl-sulphone (DDS) monotherapy. Case histories were examined for 33 patients with lepromatous leprosy (LL), diagnosed 7-48 years before the relapse and treated only with DDS during 4 to 38 years. Twenty-eight patients received irregular non-supervised polychemotherapy (PCT) since 1983. Five patients received only DDS, and presented relapses 13-20 years after the treatment was stopped. Relapses were diagnosed by clinical methods, including the reappearance of lesions or presence of new anesthetic areas. All cases were confirmed by bacilloscopy, and a subset of 20 cases by skin biopsy. Four patients presented indeterminate leprosy (IL) and one patient borderline tuberculoid leprosy (BT) in the biopsy. The latter 5 demonstrated presence of intraneural bacilli; the remainder were LL. Two patients relapsed even with PCT treatment. The others were cured with supervised PCT. Predisposing factors for relapses were as follows: DDS monotherapy, irregular PCT with inadequate dosage, unsupervised treatment, treatment uncompliance, and inadequate relationship between the patient and the health staff. Inspections for relapse in leprosy is recommended for in all multibacillary patients that were treated with DDS. The clinical appearance of new lesions or new anesthetic zones, the bacilloscopy and skin biopsy, used together, are effective in establishing the presence of relapses.

Key words: multibacillary leprosy, leprosy relapses. 
La quimioterapia de la lepra comenzó en 1941 con derivados de las sulfonas (1). La resistencia bacteriana a esta droga hizo que la Organización Mundial de la Salud (OMS) recomendara en 1982 la terapia combinada con diamino-difenil-sulfona (DDS), rifampicina (RMP) y clofazimina (CLO) durante 2 años para los pacientes multibacilares (MB) y con RMP y DDS durante 6 meses para las paucibacilares (PB) (2). Esta poliquimioterapia (PQT) ha permitido curar unos 12 millones de enfermos de lepra e impedir entre 2 a 3 millones de discapacidades $(3,4)$; se considera como un avance significativo de la medicina para el bienestar del hombre; ha permitido una reducción del $90 \%$ de la prevalencia mundial de la enfermedad, que era de 12 por 10.000 habitantes en 1985, a 1,2 por 10.000 habitantes en el 2000 (5). Basada en este éxito, la OMS propuso en 1991 la política de 'eliminación global de la lepra como problema de salud pública', que debía alcanzarse en el 2000 (6), pero se pospuso hasta el 2005 debido a que en los 27 países con mayor endemia de lepra, la incidencia no descendió entre 1985 y 1999; en los 6 países que suman el $88 \%$ de casos nuevos, el número e incidencia de nuevos casos se incrementó y el $15 \%$ de los casos nuevos correspondió a niños, lo cual indica que la transmisión activa persiste $(5,6)$.

El porcentaje de recidivas postratamiento ha sido estimado por la OMS en $0,1 \%$ por año, en pacientes MB que recibieron 24 dosis de PQT (7) y que fueron seguidos por pocos años. Los reportes del Instituto Marchoux en Bakamo registraron inicialmente recaídas de 2,9\% ० 0,8\% por 100 pacientes-año en pacientes MB tratados durante 2 años y seguidos durante $41,9 \pm 12,1$ meses (8). Cuando el seguimiento se prolongó por 2,5 años más, las recidivas subieron al $20 \%$ o 3,3 por 100 pacientes-año, recurrencias consideradas alarmantes (9). Las recidivas ocurrieron entre el tercer y el octavo año de

Correspondencia:

Gerzaín Rodríguez, Laboratorio de Patología, Instituto Nacional de Salud, CAN, Avenida Calle 26 No. 51-60, Bogotá, D.C., Colombia.

Teléfono: (571) 220 7700; fax: (571) 2200901

grodríguez@ins.gov.co

Recibido: 20/11/03; aceptado: 27/05/04 haberse suspendido la PQT y se relacionaron con una carga bacilar alta, 4 en la escala de Ridley $(9,10)$, al comienzo del tratamiento y, también, con índice bacilar (IB) alto aunque menor, al final del mismo, luego de 2 años de PQT (9).

En Colombia no existe información sobre el índice de recidivas postratamiento, aunque ha sido observada por médicos e instituciones dedicadas al manejo de la lepra. En el presente trabajo presentamos las características clínicas, bacteriológicas, terapéuticas e histopatológicas de 33 pacientes diagnosticados inicialmente con lepra lepromatosa del Sanatorio de Agua de Dios (Cundinamarca) que presentaron recidivas de su enfermedad.

\section{Materiales y métodos}

Desde 1999 llamó la atención en el Sanatorio de Agua de Dios la consulta de enfermos de lepra considerados como curados, que acudieron por presentar nuevas lesiones cutáneas con alteración de la sensibilidad, interpretadas clínicamente como recidivas de la enfermedad. También se encontraron lesiones clínicas sospechosas de recidivas en pacientes que consultaron por otros motivos.

Se realizó un estudio retrospectivo, descriptivo, de los pacientes que consultaron entre 1999 y el 2002 por los motivos mencionados anteriormente; se les hizo una revisión de la historia clínica disponible y un examen médico detallado, con exploración de la sensibilidad térmica, táctil y dolorosa y palpación de los troncos nerviosos; a todos se les practicó baciloscopia de las lesiones nuevas, del moco nasal y de los lóbulos auriculares. En 25 pacientes se realizaron una o varias biopsias de piel que se procesaron con tinciones de hematoxilina-eosina (HE), Fite-Faraco (FF) e inmunohistoquímica (IHQ) para la proteína S100. Se asumieron como criterios de inclusión para recidiva de la enfermedad, el antecedente de diagnóstico y tratamiento de lepra lepromatosa, el cuadro clínico de lepra activa, la baciloscopia de moco y linfa positiva y, en la mayoría de los casos, las lesiones de lepra en la biopsia de piel. Se consideró caso de recidiva el que cumpliera con el primer criterio y cualquiera de los 3 criterios restantes. 
De cada paciente, se registraron en una base de datos de Epiinfo, las siguientes variables: sexo, edad actual, edad al diagnóstico, años con monoterapia con DDS y con PQT, tiempo entre la suspensión del tratamiento y la recidiva, índice bacilar actual, tipo de lepra en el momento de la recidiva, tiempo con la nueva PQT y evolución de la recidiva con nueva PQT.

\section{Resultados}

Los pacientes tenían edades entre 39 y 76 años con una media de 57,5 años; el comienzo de su enfermedad con un rango entre 7 y 48 años antes de la recidiva (cuadro 1); habían sido dados de alta, bien por ausencia de lesiones clínicas activas de lepra o porque presentaron baciloscopia negativa. Todos fueron diagnosticados en el comienzo de su enfermedad como pacientes con lepra lepromatosa (LL), con baciloscopia positiva, sin gradación del IB, criterio que no se utilizaba cuando se hizo su diagnóstico inicial de lepra. Todos fueron tratados inicialmente con monoterapia con DDS, según la norma de la época que recomendaba la terapia vitalicia con esta droga, la cual recibieron durante 4 a 38 años. A partir de los años 80 , según las recomendaciones de la OMS (2), veintiocho de estos enfermos recibieron PQT con DDS, RMP y CLO, irregular, sin supervisión, en dosis variables, a veces durante varios años, según el criterio individual de los médicos tratantes, afirmación sustentada en la revisión de las historias clínicas. Además, se pudo constatar que la mayoría tuvo amplios periodos de abandono de su PQT. En resumen, ninguno de ellos recibió una PQT regular, estricta y supervisada. Cinco pacientes sólo tuvieron monoterapia con DDS y su recidiva de lepra se presentó entre 13 y 20 años después de haber dejado de tomar la DDS.

Las recidivas se diagnosticaron clínicamente en 30 de los 33 pacientes por aparición de lesiones nuevas y en 3 por aumento de la anestesia sin lesiones aparentes; se confirmó por la baciloscopia en todos los casos que reveló IB altos entre 1 y 3 , según la escala del programa colombiano de control de la lepra (11) (cuadro 2). Las biopsias de 25 enfermos confirmaron las recidivas en 20 de ellos.
Se descartó la presencia de reacción tipo 1 en estos pacientes por las características del cuadro clínico, de instauración lenta y paulatina, por la ausencia de neuritis aguda, porque no hubo exacerbación de lesiones preexistentes que nunca fueron placas calientes, edematosas o descamativas y por el estudio histopatólogico que no mostró en ninguno de los casos evidencias de esta reacción (12).

El tiempo entre la curación clínica aparente, respaldada con baciloscopias negativas, y el diagnóstico de recidivas fue en promedio de 10 años, con variaciones entre 1 y 20 años; estos datos son de difícil precisión porque no siempre se tuvo la información sobre la baciloscopia cuando el paciente se consideró curado y por la notoria irregularidad en la toma de la PQT, que se administró sin control adecuado, inclusive durante varios años.

Las biopsias se tomaron de las lesiones aparentemente nuevas o de áreas con aumento de la anestesia. En cinco pacientes mostraron infiltrados extensos, importantes, de macrófagos vacuolados, sin presencia de bacilos. Los nervios fueron difíciles de demostrar, incluso con la IHQ para $\mathrm{S} 100$ o estuvieron esclerosados. Estas biopsias se consideraron como lesiones residuales, sin signos de reactivación. En quince pacientes presentaron infiltración dérmica nodular - difusa de macrófagos vacuolados con abundantes linfocitos y plasmocitos; los nervios estaban permeados por el infiltrado y exhibían engrosamiento concéntrico perineural. El FF reveló abundantes bacilos en los infiltrados y en los nervios, con pocas formas granulosas. Los IB estuvieron entre 2 y 5 en la escala de Ridley (10) (cuadro 2). En 4 pacientes, las biopsias de las recidivas presentaron características de lepra indeterminada (LI) pero con bacilos intraneurales y, en uno, la recidiva mostró histopatología de lepra dimorfa tuberculoide (LDT) pero con presencia de bacilos enteros intraneurales con IB de 1-2 en la escala de Ridley.

En un paciente que consultó por una masa en el cuello, que correspondió a un bocio, y a quien se le practicó baciloscopia de control, se encontró un IB alto de 2,2 sin lesiones clínicas aparentes, 
Cuadro 1. Recidivas en 33 pacientes con lepra lepromatosa.

\begin{tabular}{|c|c|c|c|c|c|c|c|}
\hline Paciente & Sexo & $\begin{array}{l}\text { Edad } \\
\text { (años) }\end{array}$ & $\begin{array}{l}\text { Edad al Dx* } \\
\text { (años) }\end{array}$ & $\begin{array}{c}\text { DDS } \\
\text { (años) }\end{array}$ & $\begin{array}{l}\text { PQT irregular } \\
\text { (años) }\end{array}$ & $\begin{array}{l}\text { Fin tratamiento/ } \\
\text { recidiva** } \\
\text { (años) }\end{array}$ & $\begin{array}{c}\text { Recidiva } \\
\text { Tipo de lepra }\end{array}$ \\
\hline 1 & M & 56 & 25 & 11 & 6 & 10 & $L L^{* \star *}$ \\
\hline 2 & M & 64 & 20 & 32 & 2 & 14 & LL \\
\hline 3 & $\mathrm{~F}$ & 76 & 29 & 31 & 6 & 9 & LL \\
\hline 4 & $\mathrm{M}$ & 56 & 12 & 23 & 7 & 9 & $\mathrm{LDL}^{* * *}$ \\
\hline 5 & $\mathrm{~F}$ & 54 & 15 & 4 & 6 & 10 & $\mathrm{LL}^{* * *}$ \\
\hline 6 & $\mathrm{M}$ & 55 & 14 & 27 & 11 & 3 & $\mathrm{LL}^{* \star \star}$ \\
\hline 7 & $\mathrm{~F}$ & 64 & 24 & 19 & 5 & 10 & LL \\
\hline 8 & $\mathrm{M}$ & 64 & 26 & 21 & 11 & 6 & $\mathrm{LDT}^{\star \star \star}$ \\
\hline 9 & $\mathrm{~F}$ & 50 & 19 & 11 & 0,5 & 5 & LL \\
\hline 10 & $M$ & 44 & 16 & 9 & 9 & 9 & $\mathrm{LDL}^{* * *}$ \\
\hline 11 & $\mathrm{~F}$ & 68 & 26 & 27 & 7 & 13 & LL \\
\hline 12 & $\mathrm{M}$ & 47 & 10 & 17 & 1 & 6 & $\mathrm{LL}^{* \star *}$ \\
\hline 13 & $\mathrm{M}$ & 68 & 25 & 26 & 2 & 6 & LL \\
\hline 14 & $\mathrm{M}$ & 73 & 37 & 15 & 9 & 10 & LL \\
\hline 15 & $\mathrm{M}$ & 76 & 27 & 18 & 4 & 15 & LL \\
\hline $16^{\star \star \star \star *}$ & $M$ & 69 & 27 & 21 & 8 & 2 & $\mathrm{LL}^{* \star \star}$ \\
\hline $17^{* \star \star *}$ & $\mathrm{~F}$ & 39 & 10 & 4 & 6 & 3 & $\mathrm{LL}^{\star \star \star}$ \\
\hline 18 & $\mathrm{M}$ & 54 & 10 & 22 & 14 & 1 & $\mathrm{LL}^{* * *}$ \\
\hline 19 & $\mathrm{M}$ & 60 & 17 & 12 & 5 & 7 & $\mathrm{LL}^{* \star \star}$ \\
\hline 20 & $\mathrm{M}$ & 64 & 42 & 13 & 1 & 4 & $L L^{* * *}$ \\
\hline 21 & $\mathrm{M}$ & 68 & 32 & 4 & 4 & 12 & $L L^{* * *}$ \\
\hline 22 & $\mathrm{~F}$ & 57 & 13 & 11 & No & No & $\mathrm{LI}^{* * *}$ \\
\hline 23 & $\mathrm{~F}$ & 65 & 16 & 20 & 2 & 18 & LL \\
\hline 24 & $\mathrm{M}$ & 51 & 48 & 0 & 2 & 1 & LL \\
\hline 25 & $M$ & 71 & 26 & 25 & 4 & 16 & LL \\
\hline 26 & $\mathrm{M}$ & 57 & 13 & 5 & 1 & 13 & $\mathrm{LDL}^{\star * *}$ \\
\hline 27 & $\mathrm{~F}$ & 65 & 14 & 38 & No & 13 & $\mathrm{Ll}^{\star * *}$ \\
\hline 28 & $\mathrm{~F}$ & 48 & 11 & 18 & No & 20 & $\mathrm{LDL}^{* * *}$ \\
\hline 29 & $F$ & 56 & 23 & 17 & 1 & 16 & $\mathrm{LI}^{* * *}$ \\
\hline 30 & $\mathrm{~F}$ & 51 & 22 & 15 & No & 14 & $\operatorname{LDL}^{\star \star \star}$ \\
\hline 31 & $\mathrm{M}$ & 62 & 22 & 30 & No & 13 & LL \\
\hline 32 & $\mathrm{~F}$ & 51 & 7 & 30 & 2 & 13 & $\mathrm{Ll}^{\star \star * \star}$ \\
\hline 33 & $M$ & 49 & 18 & 7 & 2 & 18 & LL \\
\hline
\end{tabular}

* Edad al diagnóstico inicial de LL

** Años entre la suspensión del último tratamiento y la recidiva.

*** Paciente con diagnóstico histopatológico

**** Paciente que presentó 3 recidivas

DDS Diamino-difenil-sulfona

PQT Poliquimioterapia

LL Lepra lepromatosa

LDL Lepra dimorfa lepromatosa

LDT Lepra dimorfa tuberculoide

LI Lepra indeterminada

excepto por disestesias en el cuello. La biopsia de piel confirmó la existencia de lepra dimorfa lepromatosa (LDL) subclínica.

Dos enfermos presentaron dos episodios de lesiones nuevas y dos presentaron tres con las características de haber recibido monoterapia y luego PQT irregular, no supervisada y con periodos de abandono. Uno de los pacientes con 3 aparentes recidivas recibió su última $\mathrm{PQT}$ con supervisión adecuada, pero recidivó con baciloscopia positiva y con presencia de lepromas aplanados, histioides, en ambas rodillas. Creemos que tiene multirresistencia bacilar a la DDS y a la RMP, situación que se debe demostrar por inoculación en la almohadilla plantar del ratón o por biología molecular, métodos que no están 
Cuadro 2. Lepra. Indice bacilar (Ridley).

\begin{tabular}{cc}
\hline Indice bacilar & $\begin{array}{c}\text { Concentración de baar por } \\
\text { campo promedio de inmersión }\end{array}$ \\
\hline 0 & Ninguno en 100 campos \\
1 & De 1 a 10 por 100 campos \\
2 & De 1 a 10 por 10 campos \\
3 & De 1 a 10 por campo \\
4 & De 10 a 100 por campo \\
5 & De 100 a 1000 por campo \\
6 & Más de 1000 por campo \\
\hline
\end{tabular}

Cuadro 3. índice bacilar empleado en Colombia.

Cinco muestras

- (-) : no se observan baar /100 campos

- (+) : menos de 1 baar / 100 campos

- (++) : entre 1 - 10 baar / campo - 50 campos

- $(+++):>10$ baar / campo, 20 campos

- $\mathrm{IB}=$ promedio de sumatoria de + de las 5 muestras

accesibles para los autores. La otra paciente con la tercera recidiva murió por muerte violenta cuando estaba en nueva terapia PQT. En estos pacientes, ni la clínica, ni las biopsias sugieren que tuvieran reacción tipo 1 . En la paciente fallecida se documentaron clínica e histológicamente episodios de reacción tipo 2 . El segundo paciente con la tercera recidiva documentada clínicamente por baciloscopia y por biopsias repetidas, ha respondido muy bien a la nueva terapia con ofloxacina, minociclina y clofazimine.

\section{Discusión}

Agua de Dios es un municipio fundado en 1870 por enfermos de lepra, desplazados violentamente desde Tocaima, localidad vecina en donde residían; luego se adaptó como leprocomio para recibir a los enfermos de lepra de todo el país hasta que esta práctica fue abolida en 1961 (13); llegó a albergar miles de enfermos, de los cuales existen cerca de 5.000 historias clínicas en los archivos de la Institución, todos los cuales se trataban médicamente con sulfonas, a partir de 1945. En la actualidad, el Sanatorio alberga 300 pacientes de edad avanzada, con secuelas de lepra, y en el municipio, que cuenta con 14.000 habitantes, residen 1.100 que fueron enfermos de lepra, según los archivos de la institución.

Las recidivas en la lepra multibacilar se determinan con varios criterios $(8,9)$ :
1) Aumento del IB en un logaritmo de 2 en la escala de Ridley, en relación con el que tenía el paciente al darse de alta. La sola positividad no es suficiente porque algunos enfermos al terminar sus dos años de PQT pueden tener baciloscopia positiva.

2) Presencia de lesiones nuevas identificables clínicamente con las características de la lepra.

3) Presencia de bacilos viables demostrables por la inoculación a la almohadilla plantar del ratón.

Los criterios que nos permitieron establecer que estos pacientes tuvieron recidivas de lepra fueron:

1) Clínica: presencia de manchas, pápulas, placas o nódulos nuevos, con alteración de la sensibilidad, hallazgos que estuvieron presentes en 30 de los 33 pacientes; también ocurrió aumento de las zonas de anestesia o aparición de nuevas zonas anestésicas, sin lesiones clínicas aparentes, cambio que estuvo presente en 3 pacientes.

2) Baciloscopia positiva con bacilos enteros, con un índice bacilar de 2 a 3 de acuerdo con la escala usada en Colombia (11) (cuadro 3), criterio que estuvo presente en todos los casos. En tres pacientes se demostró la recidiva de la lepra a la baciloscopia, sin presencia de lesiones clínicas aparentes. Esta observación es importante porque confirma que la baciloscopia positiva como criterio de recidiva precede en meses o años a la aparición de las lesiones clínicas $(8,9)$ y es, por lo tanto, la forma más efectiva de buscar recidivas precoces. Treinta de nuestros enfermos se diagnosticaron con recidiva de la lepra por la presencia de lesiones clínicas nuevas, hecho que indica una larga evolución de la recidiva.

3) El tercer criterio que se utilizó fue el de la biopsia de piel que para ser considerada como recidiva debía mostrar inflamación intraneural o presencia de bacilos enteros en los nervios o en los macrófagos; la presencia de macrófagos espumosos sin bacilos o con escasas formas granulosas se interpretó como lesión residual y no como recidiva. De los 25 pacientes con biopsia, 15 presentaron LL, 
claramente positivos para recidiva; 5 mostraron lesiones residuales en múltiples biopsias; en 4 la imagen fue de LI y en uno de LDT. Es posible que estas características se presenten en las recidivas de la lepra $(14,15)$, como si el paciente iniciara de nuevo todo el proceso de su enfermedad, luego de un periodo de incubación que es el intervalo entre la suspensión de la PQT y la recidiva, clínica o bacteriológica. La LI de recidiva se ha llamado 'lepra indeterminada secundaria'.

En algunas lesiones, las biopsias sólo mostraron los remanentes inflamatorios de una LL tratada por lo cual es conveniente tener especímenes de varios sitios. La lepra no se reactiva globalmente sino en focos aislados que, a veces, constituyen una sorpresa. Una biopsia de piel sin cambios de lepra, o con signos residuales de la enfermedad, no excluye que el paciente tenga recidiva. Por esta razón, el manejo en conjunto de los criterios de recidiva es muy importante y cualquiera de ellos que sea positivo es suficiente para considerar que hay una recurrencia de la enfermedad.

Las recidivas en la lepra pueden ser secundarias a la persistencia de Mycobacterium leprae viables en los nervios, los ganglios linfáticos o en la piel, a $M$. leprae resistente a la monoterapia o a reinfección $(16,17)$, factor éste muy difícil de diferenciar de aquél de la persistencia bacilar. Todas estas condiciones pudieron estar involucradas en las recidivas de nuestros enfermos. Todos nuestros pacientes fueron enfermos de LL tratados con DDS durante muchos años, algunos hasta por 38. Es muy posible que los bacilos de Hansen presentes en estos enfermos fueran resistentes a la DDS y, entonces, su PQT se basaba sólo en la RMP y el CLO. Además de estas etiologías, creemos que otros factores predisponentes para la recidiva fueron la terapia irregular con dosis inadecuadas, los abandonos de la PQT, la falta de supervisión de ésta y la relación inadecuada entre el paciente y el personal de salud.

Todos estos pacientes con recidiva se sometieron a PQT según los esquemas de la OMS (2), con supervisión estricta de la ingestión del medicamento por personal de enfermería competente y comprometido, con involución satisfactoria de la recidiva por criterios clínicos y de baciloscopia y son objeto de un programa de seguimiento estricto que ya lleva 3 años para algunos enfermos, sin que se hayan observado nuevas recaídas, excepto por los dos pacientes con la tercera recidiva ya comentada, en uno de los cuales la supervisión de la toma del medicamento fue difícil por varios factores, entre ellos, el alcoholismo de la paciente. El personal de salud actual es consciente de que la administración negligente del tratamiento es una falta de ética que contribuye a crear la resistencia del bacilo a la PQT y que la aparición de cepas multirresistentes sería una eventualidad catastrófica.

Como consecuencia de la detección de estos enfermos, en la actualidad se adelanta en el Sanatorio un proyecto para detectar recidivas clínicas y subclínicas en los 1.100 pacientes MB y PB tratados y residentes en el municipio. Creemos que esta actividad debe desarrollarse en todos los pacientes que recibieron monoterapia con DDS en el país como tratamiento fundamental para la lepra, como sucedió con los enfermos del municipio de Contratación, otro antiguo lazareto de Colombia.

\section{Agradecimientos}

A Tiberio Rodríguez, auxiliar técnico del Programa de Lepra de Agua de Dios por su apoyo entusiasta y decidido en la búsqueda de las historias clínicas $\mathrm{y}$ de los pacientes.

\section{Referencias}

1. Faget GH, Pogge RC, Johansen FA, Ross $H$. Sulfanilamide in the treatment of leprosy. Public Health Rep 1942;57:1892-9.

2. WHO Study Group. Chemotherapy of leprosy for control programs. Tech Rep Ser 675. Geneva, Switzerland: WHO, 1982.

3. Word Health Organization. Elimination of leprosy. Status Report. Geneva, Switzerland: WHO; 1999. www.who.int/lep.

4. Rodríguez G. Editorial. La eliminación de la lepra. Biomédica 2001;21:1-3.

5. Word Health Organization. A global alliance for leprosy elimination. Strategic plan, 2000-2005. Geneva Switzerland: WHO; 1999. www.who.int/lep.

6. Lockwood D. Leprosy elimination a virtual phenomenon or a reality? Br Med J 2002;324:1516-8. 
7. ALM Consensus Development Conference on the Chemotherapy of Leprosy. Consensus development statement on the chemotherapy of leprosy. Int J Lepr 1992;60:644-52.

8. Marchoux Chemotherapy Study Group. Relapses in multibacillary leprosy patients after stoping treatment with rifampin-containing combined regimens. Int $\mathrm{J}$ Lepros 1992;60:525-35.

9. Jamet P, Ji B, Marchoux Chemotherapy Study Group. Relapse after long-term follow up of multibacillary patients treated by WHO multidrug regimen. Int J Lepr Other Micobact Dis 1995;63:195-201.

10. Ridley DS, Hilson GRF. A logarithmic index of bacilli in biopsies. A. Method. Int J Lepros 1967;25:184-6.

11. Ministerio de Salud. Guía de atención de la lepra. En: Normas técnicas y guías de atención. Tomo II. Bogotá: Ministerio de Salud; 2000.
12. Rodríguez G, Orozco LC. Lepra. Bogotá: Instituto Nacional de Salud; 1996.

13. Velandia R. Historia documentada de la Fundación de Agua de Dios. Bogotá: Editora Guadalupe; 2002.

14. Ramu G. The past, present and future of chemotherapy of leprosy. NLO Bull 1994;23:37-49.

15. Waters MFR, Ridley DS. Tuberculoid relapse in lepromatous patients. Int J Lepros 1979;47:350.

16. Shetty VP, Sucritra K, Uplekar MW, Antia NH. Higher incidence of viable Mycobacterium leprae within the nerve as compared to skin among multibacillary leprosy patients released from multidrug therapy. Lepr Rev 1997;68:131-8.

17. Sharma A, Sharma VK, Rajwanshi A, Das A, Kaur I, Kumar B. Presence of $M$. leprae in tissues in slit skin smear negative multibacillary (MB) patients after WHOMBR. Lepr Rev 1999;70-281-6. 